\title{
Wheal and flare reactions in skin prick tests of patients treated with montelukast alone or in combination with antihistamines
}

\author{
Malgorzata Gorska Ciebiada • Marcin Barylski • \\ Maciej Ciebiada
}

Received: 11 June 2013/Revised: 6 November 2013/ Accepted: 19 November 2013/Published online: 27 November 2013

(C) The Author(s) 2013. This article is published with open access at Springerlink.com

\begin{abstract}
Background Because antileukotrienes may inhibit inflammation, it is plausible that montelukast administered for a long time could suppress skin wheal and flare reaction, and thus, it should be discarded prior to the tests. This study assessed the effect of long-lasting treatment with montelukast alone or in combination with antihistamines on wheal and flare in skin pricks tests (SPT) in patients sensitized to perennial allergens.

Methods We conducted a 32-week, double-blind, placebo-controlled, cross-over and randomized trial that implicated two arms: arm A, 20 patients received levocetirizine, montelukast with or without levocetirizine or placebo; arm B, 20 patients received desloratadine, montelukast with or without desloratadine or placebo. All treatment periods lasted 6 weeks and were separated by 2-week washouts. At baseline and on the last day of each treatment period, SPT were performed in all participants.
\end{abstract}

Responsible Editor: Bernhard Gibbs.

Pharmaceutical companies had no input into the design or implementation of this study.

M. G. Ciebiada

Department of Internal Medicine and Diabetology, Medical

University of Lodz, Lodz, Poland

M. Barylski

Department of Internal Disease and Cardiological Rehabilitation, Medical University of Lodz, Lodz, Poland

M. Ciebiada $(\bowtie)$

Department of General and Oncological Pneumonology,

Medical University of Lodz, Kopcinskego Street 22,

90-153 Lodz, Poland

e-mail: maciej.ciebiada@umed.lodz.pl; maciej_ciebiada@op.pl
Results Both levocetirizine and desloratadine in monotherapy, or in combination with montelukast, were effective in reducing wheal and flare in SPT. Monotherapy with montelukast did not change the size of the wheal for either histamine or for house dust mites, in either arm of the study, but significantly reduced the size of flare for histamine in arm A. Addition of montelukast to antihistamine did not exceed efficacy of monotherapy with antihistamine in both arms of the study.

Conclusions Since the size of wheal determines the results of SPT, montelukast, even taken for a long time, does not have to be discarded prior to the tests.

Keywords Allergic rhinitis - Montelukast . Antihistamine $\cdot$ Skin prick test $\cdot$ Inflammation

\section{Introduction}

Skin prick tests (SPT) are commonly used to confirm sensitization to a wide spectrum of environmental allergens. SPT help to diagnose the underlying cause of rhinitis, asthma or urticaria, and are required to recommend appropriate prophylaxis or for qualification for immunotherapy $[1,2]$. Since early wheal and flare reactions result mainly from histamine released from degranulating mast cells, it is obvious that antihistamines are more or less able to inhibit this reaction [3].

Since montelukast, a potent and selective leukotriene receptor antagonist, suppresses allergic inflammation [48], improves control of asthma [8] and reduces symptoms of seasonal $[4,5]$ and perennial $[6,7]$ allergic rhinitis (AR), it is possible that it may inhibit skin response to allergens measured in skin prick tests. Although guidelines for skin prick testing do not recommend discontinuation of 
montelukast before the SPT [1, 2], most studies relied on assessment of wheal and flare reactions after the single dose [9] or a very short-term treatment with montelukast [10]. Furthermore, there were studies that confirmed gradually increasing improvement of AR symptoms in the course of long-lasting treatment with montelukast [7, 11, 12] or combination of montelukast with antihistamine [11]. Therefore, it is plausible that one dose or short treatment with montelukast may not affect SPT, whereas long lasting treatment with this agent administered alone or in combination with antihistamine due to the increasing efficacy and immunomodulative properties may affect the skin response in SPT.

In this study, we aimed to determine the influence of montelukast administered for 6 weeks as monotherapy or added to antihistamine on the size of wheal and flare in SPT of patients with allergic rhinitis sensitized to perennial allergens, in relation to placebo as well as to monotherapy with desloratadine or levocetirizine.

\section{Study design}

This study was designed as a prospective, double-blind, randomized, cross-over and controlled with placebo, including two arms with a 2-week run-in period and four treatment periods each lasting for 6 weeks separated by 2-week washouts.

Patients were recruited in our outpatient clinic over 4 months (June-September) and study was performed between September and March.

Study included male and female patients, aged 18-65 years with at least 2 years history of mild to severe persistent allergic rhinitis who were sensitized to perennial allergens relevant to Central Europe (HDM: house dust mite, cat and dog), confirmed by a positive history and positive results of skin prick tests, whereas patients who suffered from skin diseases that prevented execution and interpretation of skin prick tests, who were treated with systemic steroids or immunomodulative medicaments, as well as patients who were current smokers, with infection within 6 weeks preceding the study or with neoplasmatic disease and severe diseases, were excluded from the study. Pregnant and breast-feeding women were excluded too. Patients could not use an allergen-specific immunotherapy or any anti-allergic medications during the course of the study except the study medication. Xylomethasoline $(0.1 \%)$ nasal drops were allowed as a rescue medication.

After a two-week run-in period, all eligible patients (30 female, 10 male, mean age was $28.9 \pm 2.7)$ were assigned randomly into group A $(n=20)$ receiving either levocetirizine $(5 \mathrm{mg}$ tablet one daily in the evening) or montelukast (10 $\mathrm{mg}$ tablet once daily in the evening) or a combination of montelukast and levocetirizine (in the evening) or placebo, or to group $\mathrm{B}(n=20)$ receiving either desloratadine $(10 \mathrm{mg}$ tablet once daily in the evening) or montelukast $(10 \mathrm{mg}$ tablet once daily in the evening), or a combination of montelukast and desloratadine (in the evening) or placebo (5 $\mathrm{mg}$ saccharose in starch pills, one daily in the evening). Medications were administered in a cross-over and blinded manner.

Both at baseline and on the last day of each treatment period, skin prick tests were done for each participant.

All patients signed written informed consent and the study protocol was approved by the ethical committee of the Medical University in Lodz.

The principal endpoint of this study was the size of wheal and flare in skin prick tests after the 6 weeks of treatment, either with monotherapy with antihistamine (desloratadine or levocetirizine) and montelukast, or with combination therapy, which included antihistamine and montelukast in relation to the baseline test and placebo.

Skin prick tests

Skin prick tests with 11 common allergens (Allergopharma J. Ganzer KG, Reinbeck, Germany) were performed for each patient, with histamine $(10 \mathrm{mg} / \mathrm{ml})$ as a positive and diluent as a negative control. Results were regarded as positive when the mean wheal diameter (assessed as a sum of the largest diameter and its perpendicular measurement) was greater than or equal to $3 \mathrm{~mm}$. Since all patients were sensitized to the house dust mites, results of the SPT were presented for Dermatophagoides pteronyssinus and farinae in relation to the histamine.

\section{Statistical methods}

The distribution of the results was determined with the Shapiro-Wilk normality test, while a Mann-Whitney test was used to compare groups and one-way analysis of variance (Anova) was done to compare results in each arm on different visits. A $p<0.05$ was considered as statistically significant. The mean with standard error of the mean (SEM) was provided. Statistica 5.1 PL for Windows software (StatSoft Polska, Cracow, Poland) was used for analyses.

\section{Results}

All randomized patients completed four treatment periods, and only two patients were lost to follow-up. All participants were sensitized to house dust mites and six patients were additionally sensitized to cat and dog allergens. Although sensitization to seasonal allergens was present in 
Table 1 Patients' baseline characteristics

\begin{tabular}{lll}
\hline & $\begin{array}{l}\text { A Montelukast/ } \\
\text { levocetirizine arm }\end{array}$ & $\begin{array}{l}\text { B Montelukast/ } \\
\text { desloratadine arm }\end{array}$ \\
\hline Number of subjects & 20 & 20 \\
Mean age & $23.65 \pm 2.1$ & $34.1 \pm 2.69$ \\
Sex F:M & $14: 6$ & $16: 4$ \\
$\begin{array}{l}\text { Ethnic origin } \\
\begin{array}{c}\text { Duration of persistent } \\
\text { allergic rhinitis (years) }\end{array}\end{array}$ & Caucasian $(100 \%)$ & Caucasian $(100 \%)$ \\
$\begin{array}{c}\text { Severity of AR (according } \\
\text { to ARIA) }\end{array}$ & Moderate/severe & Moderate/severe \\
\hline
\end{tabular}

Data are expressed as mean value \pm SEM

some patients, it was not essential in relation to HDM. Patients' baseline characteristics are presented in Table 1 .

Generally, the mean size of the wheal and flare was the biggest at baseline. The placebo did not affect the size of skin reactions both to histamine and HDM in patients evaluated in the arm A and B (Table 2; Figs. 1, 2, 3).

Levocetirizine administered as monotherapy or in combination with montelukast in arm A and monotherapy with desloratadine, as well as concomitant treatment with desloratadine and montelukast in arm B were the most effective treatment options of inhibiting the size of wheal and flare in SPT. There were no significant differences between antihistamine taken alone or in combination with montelukast (Table 2; Figs. 1, 2, 3).

If montelukast was administered as monotherapy, it did not change the size of the wheal for either histamine or for HDM, in either arm of the study. However, montelukast significantly reduced the size of flare in the SPT with histamine in arm A and slightly, but not significantly $(p=0.052)$, reduced the size of flare for $D$. pteronyssinus in arm B. Administration of montelukast in combination with antihistamine had no effect on the size of wheal and flare in comparison to monotherapy with antihistamine in both arms of the study (Table 2; Figs. 1, 2, 3).

\section{Discussion}

The results of this study demonstrate that long-term therapy with montelukast, which is administered in monotherapy or concomitantly with the levocetirizine or desloratadine, does not affect formation of wheals in SPT, nor does it potentiate the inhibitory effect of antihistamines. Since the diameter of the wheal underlies the assessment of results of SPT, slight inhibition of flare by montelukast does not affect outcomes of the test; thus montelukast, even administered for a long time, does not have to be discarded before the skin prick test.

The formation of wheals and flares in skin prick tests result from an immunoglobulin E-dependent basophils and mast cells activation, marked by the release of inflammatory mediators, including histamine [1]. Histamine induces capillary dilation, increases vascular permeability, stimulates nociceptors responsible for pain, and further causes eosinophil chemotaxis to the inflamed tissue. As a result, the exudation enters the skin and causes swelling accompanied by itching [1, 2]. Although it is unlikely that montelukast, a potent leukotriene receptor antagonist,

Table 2 Flare reaction in patients treated with: montelukast alone; levocetirizine alone; placebo or with the combination of montelukast and levocetirizine (group A) and montelukast alone; desloratadine alone; placebo or with the combination of montelukast and desloratadine (group B)

\begin{tabular}{lccc}
\hline & Histamine & D. pteronyssinus & D. farinae \\
\hline A & & & \\
$\quad$ Baseline & $13.35 \pm 1.88$ & $11.2 \pm 2.71$ & $13.9 \pm 2.9$ \\
Placebo & $11.3 \pm 1.66$ & $11.25 \pm 2.79$ & $12.9 \pm 2.5$ \\
Levocetirizine & $2.05 \pm 0.67^{* *}$ & $4.6 \pm 1.35^{* *}$ & $4.75 \pm 1.67^{* *}$ \\
Montelukast & $8.95 \pm 1.65^{*}$ & $11.5 \pm 2.74$ & $14.55 \pm 2.73$ \\
Montelukast + levocetirizine & $2.75 \pm 0.58^{* *}$ & $5.15 \pm 1.89^{* *}$ & $5.5 \pm 1.84^{* *}$ \\
B & & & $10.6 \pm 2.9$ \\
Baseline & $13.1 \pm 1.53$ & $8.55 \pm 2.42$ & $9.75 \pm 2.53$ \\
Placebo & $11.65 \pm 1.73$ & $5.6 \pm 2.2^{* *}$ & $9.25 \pm 2.28$ \\
Desloratadine & $3.7 \pm 1.2^{* *}$ & $9.35 \pm 2.46$ & $4.45 \pm 1.8^{* *}$ \\
Montelukast & $13.26 \pm 1.8$ & $6.1 \pm 2.13^{* *}$ & $11.1 \pm 2.46$ \\
Montelukast + desloratadine & $4.4 \pm 1.32^{* *}$ & $5.75 \pm 2.02^{* *}$ \\
\hline
\end{tabular}

Values \pm SEM

$n$ Number of subjects

$* p<0.05$ vs. baseline

** $p<0.01$ vs. baseline, placebo and montelukast 

for histamine in patients treated in montelukast/levocetirizine arm (graph A), and montelukast/ desloratadine arm (graph B).

Data are expressed as mean \pm SEM; $\mathrm{m}+1$, montelukast with levocetirizine; $\mathrm{m}+\mathrm{d}$, montelukast with desloratadine; ${ }^{* *} p<0.01$
Fig. 1 Mean diameter of wheal
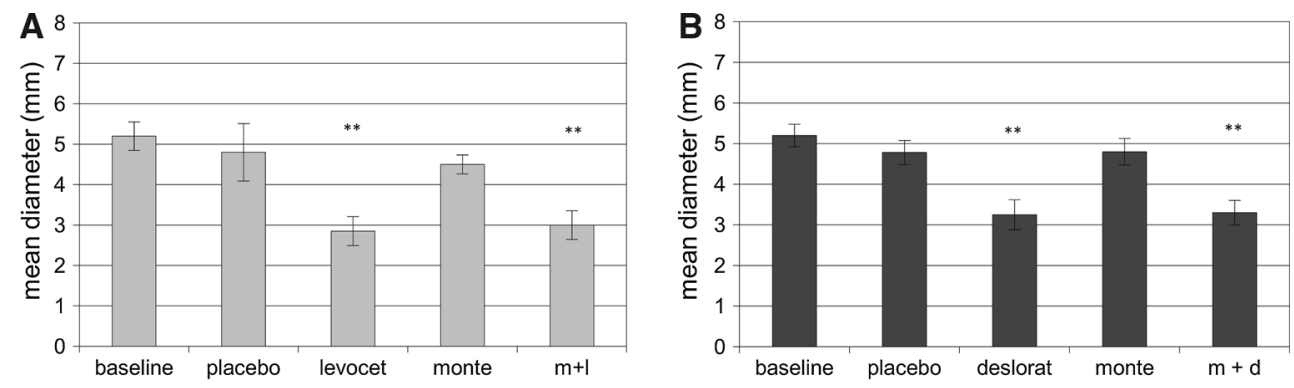

Fig. 2 Mean diameter of wheal for Dermatophagoides pteronyssinus in patients treated in montelukast/levocetirizine arm (graph A), and montelukast/ desloratadine arm (graph B).

Data are expressed as mean $\pm \mathrm{SEM} ; \mathrm{m}+1$, montelukast with levocetirizine; $\mathrm{m}+\mathrm{d}$, montelukast with desloratadine; $* * p<0.01$
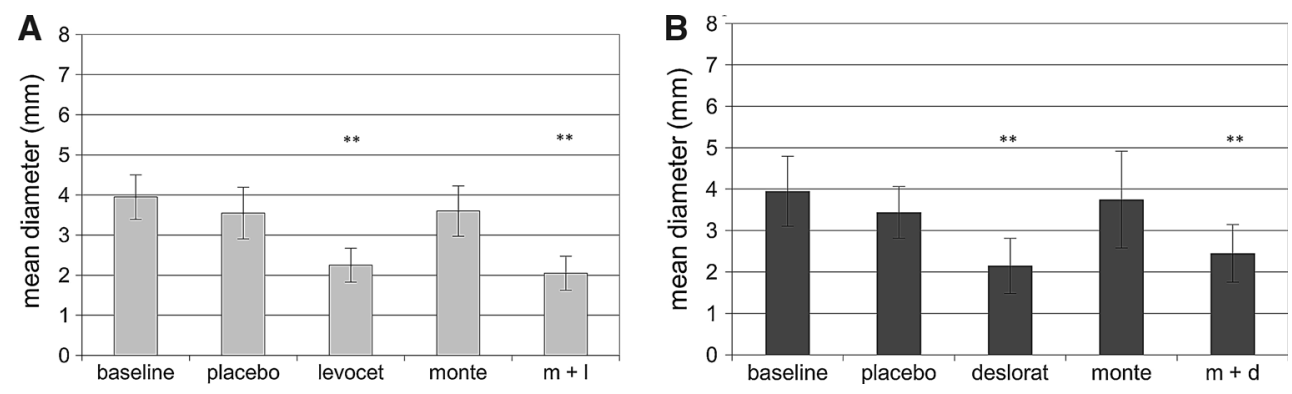

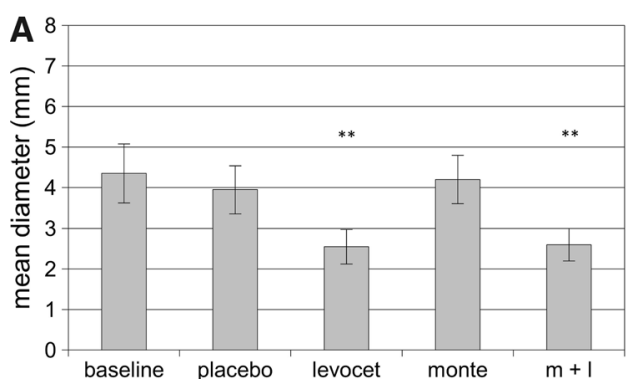

Fig. 3 Mean diameter of wheal for Dermatophagoides farinae SPT in patients treated in montelukast/levocetirizine arm (graph A), and montelukast/desloratadine arm (graph B). Data are expressed as

directly affects release of histamine from basophils and mast cells [10,13], it is well documented that it possesses other anti-inflammatory properties [14-18], and may increase the clinical effect during the treatment $[11,12]$. Thus, if taken for a long time, it could alter the inflammatory response of the skin.

Montelukast has been able to modify the skin response; however, the number of studies supporting this finding is still obscure. In rats exposed to water avoidance stress, 5-day treatment with montelukast decreased the number of both degranulated and mature granulated mast cells in the dermis [19]. In humans, montelukast significantly delayed the occurrence of late skin responses, which constitute very frequent side effects of specific immunotherapy [9], and decreased the severity of severe hypersensitivity reactions to platinum in patients undergoing rapid desensitization [20]. In patient with delayed pressure urticaria, addition of montelukast to antihistamine resulted in better

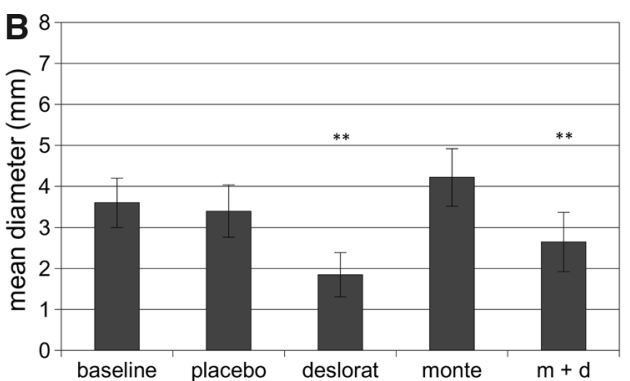

mean $\pm \mathrm{SEM} ; \mathrm{m}+1$, montelukast with levocetirizine; $\mathrm{m}+\mathrm{d}$, montelukast with desloratadine; ${ }^{* *} p<0.01$

improvement regarding the suppression of the challenge test and clinical improvement [21].

Despite its anti-inflammatory properties and clinical efficacy, a single dose [9] or 1-week treatment with montelukast $[10,22]$ did not significantly suppress both wheal $[9,10,22]$ and flare $[9,10]$ at any time point compared with placebo. Furthermore, in this field, the efficacy of short treatment with montelukast was always inferior to efficacy of antihistamines [9, 10]. What is more, montelukast as add-on therapy to antihistamine did not bring any additional benefits compared to monotherapy with antihistamine [10].

Similarly in this study, the long-lasting treatment with montelukast did not alter the size of the wheal in SPT. The efficacy of montelukast was comparable to placebo, and significantly lower than efficacy of monotherapy with levocetirizine and desloratadine in $\operatorname{arm} \mathrm{A}$ and $\mathrm{B}$, respectively. Furthermore, montelukast did not change the mean 
diameter of flare for HDM in arm A and B and histamine in arm $\mathrm{B}$, but significantly reduced the size of the flare to histamine in arm A when compared to baseline. However, the efficacy of montelukast was lower than the efficacy of levocetirizine and desloratadine and comparable to placebo at any time point of the study. Also, addition of montelukast did not potentiate effects of antihistamines.

The results of our study confirm that montelukast, which may modify tissue infiltration and inflammatory milieu, and subsequently may modify the late phase of allergic inflammation, does not seem to affect release of histamine from activated mast cells and basophils. Therefore, montelukast, even if is taken for a long time, does not need to be discontinued before allergen skin testing. The reduction of flare for histamine in patients treated with montelukast results from a small number of participants, rather than anti-inflammatory properties of montelukast, and does not affect reading of SPT where the mean size of the wheal is important.

Acknowledgments The study was self-funded (Medical University of Lodz).

Conflict of interest The authors have no conflicts of interest or competing financial interests with regards to this manuscript.

Open Access This article is distributed under the terms of the Creative Commons Attribution License which permits any use, distribution, and reproduction in any medium, provided the original author(s) and the source are credited.

\section{References}

1. Bousquet J, Heinzerling L, Bachert C, et al. Practical guide to skin prick tests in allergy to aeroallergens. Allergy. 2012; 67(1):18-24.

2. Bousquet J, Schünemann HJ, Samolinski B, et al. Allergic rhinitis and its impact on asthma (ARIA): achievements in 10 years and future needs. J Allergy Clin Immunol. 2012;130(5):1049-62.

3. dos Santos RV, Magerl M, Mlynek A, et al. Suppression of histamine- and allergen-induced skin reactions: comparison of first- and second-generation antihistamines. Ann Allergy Asthma Immunol. 2009;102(6):495-9.

4. Nayak AS, Philip G, Lu S, et al. Efficacy and tolerability of montelukast alone or in combination with loratadine in seasonal allergic rhinitis: a multicenter, randomized, double blind, placebo controlled trial performed in the fall. Ann Allergy Asthma Immunol. 2002;88(6):592-600.

5. Meltzer EO, Malmstrom K, Lu S, et al. Concomitant montelukast and loratadine as treatment for seasonal allergic rhinitis: a randomized, placebo controlled clinical trial. J Allergy Clin Immunol. 2000;105(5):917-22.
6. Ciebiada M, Gorska-Ciebiada M, DuBuske LM, et al. Montelukast with desloratadine or levocetirizine for the treatment of persistent allergic rhinitis. Ann Allergy Asthma Immunol. 2006;97(5):664-71.

7. Patel P, Philip G, Yang W, et al. Randomized, double-blind, placebo-controlled study of montelukast for treating perennial allergic rhinitis. Ann Allergy Asthma Immunol. 2005;95(6): $551-7$.

8. Amlani S, Nadarajah T, McIvor RA. Montelukast for the treatment of asthma in the adult population. Expert Opin Pharmacother. 2011;12(13):2119-28.

9. White M, Rothrock S, Meeves S, et al. Comparative effects of fexofenadine and montelukast on allergen-induced wheal and flare. Allergy Asthma Proc. 2005;26(3):221-8.

10. Simons FE, Johnston L, Gu X, et al. Suppression of the early and late cutaneous allergic responses using fexofenadine and montelukast. Ann Allergy Asthma Immunol. 2001;86(1):44-50.

11. Ciebiada M, Gorska-Ciebiada M, Barylski M, et al. Use of montelukast alone or in combination with desloratadine or levocetirizine in patients with persistent allergic rhinitis. Am J Rhinol Allergy. 2011;25(1):1-6.

12. van Adelsberg J, Philip G, Pedinoff AJ, et al. Montelukast improves symptoms of seasonal allergic rhinitis over a 4 week treatment period. Allergy. 2003;58(12):1268-76.

13. Sade K, Kivity S, Fireman E, et al. Effect of montelukast on basophil releasability in patients with asthma. Isr Med Assoc J. 2005;7(12):792-5.

14. Hallstrand TS, Moody MW, Wurfel MM, et al. Inflammatory basis of exercise-induced bronchoconstriction. Am J Respir Crit Care Med. 2005;172(6):679-86.

15. Laitinen A, Lindqvist A, Halme $M$, et al. Leukotriene $E(4)$ induced persistent eosinophilia and airway obstruction are reversed by zafirlukast in patients with asthma. J Allergy Clin Immunol. 2005;115(2):259-65.

16. Maeba S, Ichiyama T, Ueno Y, Furukawa S, et al. Effect of montelukast on nuclear factor kappaB activation and proinflammatory molecules. Ann Allergy Asthma Immunol. 2005;94(6): $670-4$.

17. Nagata M, Saito K, Kikuchi I, et al. Effect of the cysteinyl leukotriene antagonist pranlukast on transendothelial migration of eosinophils. Int Arch Allergy Immunol. 2005;137(1):2-6.

18. Wu AY, Chik SC, Chan AW, et al. Anti-inflammatory effects of high-dose montelukast in an animal model of acute asthma. Clin Exp Allergy. 2003;33(3):359-66.

19. Cikler E, Ersoy Y, Cetinel S, et al. The leukotriene d4 receptor antagonist, montelukast, inhibits mast cell degranulation in the dermis induced by water avoidance stress. Acta Histochem. 2009;111(2):112-8.

20. Breslow RG, Caiado J, Castells MC. Acetylsalicylic acid and montelukast block mast cell mediator-related symptoms during rapid desensitization. Ann Allergy Asthma Immunol. 2009; 102(2):155-60.

21. Nettis E, Colanardi MC, Soccio AL, et al. Desloratadine in combination with montelukast suppresses the dermographometer challenge test papule, and is effective in the treatment of delayed pressure urticaria: a randomized, double-blind, placebo-controlled study. Br J Dermatol. 2006;155(6):1279-82.

22. Hill SL, Krouse JH. The effects of montelukast on intradermal wheal and flare. Otolaryngol Head Neck Surg. 2003;129(3): 199-203. 